\title{
LA CÉLÉBRATION DES QUINZE ANS : SIGNIFICATIONS ET ÉVOLUTIONS D'UN RITE DE SORTIE DE L'ENFANCE POUR LES FILlES AU PÉROU
}

\author{
Robin Cavagnoud
}

Pour les jeunes filles péruviennes, la célébration du quinzième anniversaire est un événement fondateur marquant culturellement la fin de l'enfance. Dans sa mise en scène traditionnelle, majoritaire dans les couches populaires, ce rite de passage met au centre de son déroulement la place de la famille nucléaire et élargie. Il annonce le mariage et signifie une entrée dans l'âge adulte féminin. Dans ses formes contemporaines et modernes, au contraire, il met l'accent sur la participation des groupes de pairs, au détriment de la famille et de la parenté. Les recréations actuelles de ce rite, notamment à travers les fêtes juvéniles et les voyages à l'étranger, mettent en évidence l'émergence de la catégorie de jeunesse comme étape de vie transitoire entre l'enfance et l'âge adulte. Des données démographiques corroborent cette analyse et vont dans le sens de ces observations ethnosociologiques.

Dans le champ juridique international, l'enfance prend officiellement fin à 18 ans, âge de la majorité légale : «Un enfant s'entend de tout être humain âgé de moins de dix-huit ans, sauf si la majorité est atteinte plus tôt en vertu de la législation qui lui est applicable. $\gg^{[1]}$ Cette approche formelle facilite la détermination des limites de l'enfance à partir du seul critère de l'âge ( 0 à 18 ans). Au Pérou, la majorité légale est également fixée à 18 ans mais le Code de l'enfant et de l'adolescent fait une distinction dans son article premier entre enfance et adolescence : «Est considéré comme enfant tout être humain de sa naissance à l'âge de 12 ans et adolescent tout être humain de 12 à 18 ans. $\gg^{[2]}$ Ces définitions légales renvoient au champ juridique de l'enfance et s'avèrent inadéquates pour les sciences sociales, et notamment la sociologie, qui considèrent fondamentalement l'enfance comme une construction socioculturelle ${ }^{[3]}$. Sa signification et ses limites varient en effet d'un contexte social et culturel à un autre, ce qui conduit à parler de la

[1] Onu, Unicef, Convention internationale relative aux droits de l'enfant, New York, 1989.

[2] « Se considera niño a todo ser humano desde su concepción hasta cumplir los doce años de edad y adolescente desde los doce hasta cumplir los dieciocho años de edad. » in CONGRESO DE LA REPÚBLICA, Código peruano del niño y del adolescente, Lima, 1992.

[3] Allison JAMES, Alan PROUT, Constructing and Reconstructing Childhood, London, Falmer, 1997. 
"pluralité de l'enfance »[4]. La sociologie doit donc se doter de critères autres que l'âge pour cerner dans chaque société et culture le début et la fin de l'enfance.

Dans le cas du Pérou, un rite important symbolise la sortie de l'enfance des jeunes filles : la cérémonie du quinzième anniversaire appelée quinceañera ou fiesta quinceañera. Cet événement se célèbre, avec quelques différences dans son déroulement, dans la plupart des pays d'Amérique latine et tous les milieux sociaux et culturels ${ }^{[5]}$. Cet anniversaire est plus important que les autres dans le parcours de vie des jeunes filles, car il introduit une « coupure » dans la continuité du temps par rapport à un certain ordre établi et détermine un « avant » et un « après » ${ }^{[6]}$. Considéré comme une présentation officielle à la société, il constitue un événement plus marquant encore que la communion ou la célébration de la fin du collège $\mathrm{e}^{[7]}$.

Cet article décrypte dans un premier temps la fête des 15 ans telle qu'elle se célèbre de manière traditionnelle pour les jeunes filles péruviennes comme rite de sortie de l'enfance et annonce de l'âge adulte féminin. De cette première analyse, nous étudierons ensuite les réinventions rituelles récentes de cet événement, notamment dans les classes moyennes de Lima, en soulignant les significations de ces pratiques novatrices. Enfin, une troisième partie questionnera les évolutions de ce rite par rapport à l'émergence de la catégorie de jeunesse comme étape de transition entre l'enfance et l'âge adulte. Un lien sera alors établi avec les évolutions du statut des jeunes filles dans la société péruvienne dans un contexte de modernisation avancée.

Cet article entend l'enfance comme l'une des périodes majeures de la vie, au même titre que l'âge adulte ou la vieillesse (troisème et quatrième âges). La méthode employée dans cette enquête a été l'observation participante, à diverses reprises entre 2006 et 2010, du déroulement de cette célébration à Lima. Nous avons également recueilli une trentaine de témoignages auprès de filles adultes péruviennes (de Lima dans la majorité), étudiantes et mères de familles de 15 à 45 ans, ayant connu cette fête marquant le terme de leur enfance au regard de la famille et de la sociétée ${ }^{[8]}$.

\section{$\checkmark$ La célebration des 15 ans: une cérémonie traditionnelle pour les jeunes filles péruierenes}

\section{Déroulement du rite}

La cérémonie a généralement lieu au domicile de la jeune fille ou dans une salle louée par la famille. Le recours à un groupe de musiciens ou à un disc jockey est également prévu par la famille

[4] Chris JENKS, Childhood, New York, Abingdon Routledege, 1996.

[5] Gerdien STEENBEEK, Vrouwen op de drempel. Gender en moraliteit in een Mexicaanse provinciestad, Amsterdam, Thela Publishers, 1995.

[6] Michel BERTRAND, «Penser l'événement en histoire : mise en perspective d'un retour en grâce », in Marc BESSIN, Claire BIDART, Michel GROSSETTI (dir.) Bifurcations. Les sciences sociales face aux ruptures et à l'événement, Paris, La Découverte, 2010, pp. 36-50.

[7] Jacobijn OLTHOFF, A Dream Denied. Teenage Girls in Migrant Popular Neighbourhoods, Lima, Peru, Utrecht, University of Utrecht, 2006.

[8] Les témoignages ont été obtenus à partir des questions suivantes : 1. Comment as-tu célébré ton quinzième anniversaire ? 2. Estce que ça t'a plu et est-ce que tu penses que ça a été un moment important de ta vie ? 3. As-tu senti un changement dans ta vie après cette célébration ? 4. Penses-tu que maintenant on célèbre moins le quinzième anniversaire des filles ou qu'on le célèbre différemment, c'est-à-dire avec un changement dans les coutumes ? La traduction des témoignages a été assurée par l'auteur. 
pour assurer le service musical tout au long de la célébration et de la fête qui suit. Les festivités débutent avec l'entrée de la jeune fille, maquillée et coiffée avec soin et vêtue d'une longue robe souvent blanche spécialement achetée pour l'occasion, accompagnée de son père, sur une musique lente. Les deux protagonistes entament rapidement la danse d'une première valse qui marque le point de départ officiel de la cérémonie. Autour d'eux, les amis filles et garçons de la jeune fille accompagnent la danse. Les amies sont également habillées de façon très élégante mais la couleur de leur robe, identique entre elles, doit se distinguer de celle de la quinceañera (par exemple verte ou bleue par opposition à la robe blanche ou de tons pâles de la jeune fille qui doit être la seule à porter cette couleur, symbole de pureté). À l'image du père, les garçons sont également vêtus d'un costume le plus souvent noir et d'une cravate assortie. La formalité est donc de rigueur et marque le caractère exceptionnel et solennel de la célébration. Après la première danse avec son père, la jeune fille entame une nouvelle valse avec chaque membre de la famille de sexe masculin, selon son degré de proximité : frères, oncles, grands-parents, etc. Ensuite, et dans les cérémonies les plus minutieusement préparées, une chorégraphie des meilleurs amis accompagne plusieurs chansons à la mode avec la jeune fille au centre de la scène.

La salle où se déroule la fête est décorée de ballons gonflables dont la couleur s'accorde avec la robe de la jeune fille. Elle est également entourée de nombreuses tables et chaises ainsi que de buffets où tous les invités peuvent s'alimenter librement après les premières danses et le toast. Par ailleurs, il est important de faire remarquer que dans les familles les plus attachées à la religion catholique, la célébration au domicile de la famille peut être précédée d'une messe d'action de grâces à l'église. La jeune fille arrive alors dans son habit de fête, accompagnée des membres de la famille les plus proches (parents, oncles et grands-parents) et de demoiselles d'honneur plus jeunes qu'elle (nièces ou cousines). Après la messe et avant la sortie de l'église, la jeune fille doit déposer son bouquet à la gloire de la vierge Marie. L'association peu fréquente de la célébration des 15 ans à la cérémonie à l'église montre que le rite ne revêt pas de connotation religieuse aussi marquée que la communion ou le mariage. Il dévoile une certaine liberté laissée aux familles quant aux choix ponctuant son déroulement (lieu, couleur de la robe, invités, etc.) même s'il s'agit d'une cérémonie traditionnellement liée à la religion catholique. Au Pérou, seules les filles de confession juive ou celles traversant une crise familiale ne célèbrent pas le rite du quinzième anniversaire.

Après la séquence des premières danses, chaque membre de la famillle de la jeune fille prend la parole pendant quelques minutes pour exprimer la joie ressentie à l'occasion de cet événement. Le père est le premier à parler, suivi de la mère, des frères et sœurs, oncles et grands-parents. Les amis de la jeune fille peuvent également être encouragés à s'exprimer, en particulier ses copines les plus proches, mais il s'agit-là d'une règle moins stricte. Cette prise de parole des membres de la famille est l'occasion de déclarer leur affection pour la quinceañera, la fierté de la voir grandir et d'atteindre son quinzième anniversaire, étape décisive dans son cheminement vers l'âge adulte et sa sortie de l'enfance. À l'issue de cette séquence de prise de parole est porté un toast en l'honneur de la jeune fille. Puis commence le repas, généralement un buffet installé sur des tables dans un espace de la salle. Pour plus de commodités, les familles louent généralement les services d'une entreprise de restauration à domicile (catering) pour éviter de perdre du temps dans la préparation et le service de la nourriture. Cela dépend de leurs moyens économiques mais nombre d'entre elles n'hésitent pas à investir de fortes quantités d'argent (plusieurs centaines, 
voire milliers de dollars) à cette occasion pour garantir le succès de cette célébration, non seulement au regard des membres de la famille élargie mais aussi des amis présents. Après le repas, les participants sont invités à poursuivre la fête en dansant sur des musiques plus juvéniles (salsa, hip hop, rock), moins formelles que les valses marquant la première partie de la cérémonie. Cette partie est la plus festive de la cérémonie et s'accompagne de la consommation d'alcool, pour les adultes présents et les adolescents sous le contrôle de leurs parents.

Si le déroulement de la cérémonie des 15 ans suit les différentes séquences décrites ici, des aménagements sont souvent réalisés par la famille, en fonction du nombre d'invités et du lieu de la célébration. Il s'agit de l'organisation la plus traditionnelle et commune de la cérémomie des 15 ans au Pérou, également la plus populaire et partagée par toutes les couches sociales, notamment les plus modestes.

\section{Mise en scène ritualisée d'un changement de statut}

La célébration des 15 ans est un événement prévu et programmé par la jeune fille et sa famille ${ }^{[9]}$. Elle est le plus souvent préparée plusieurs semaines, voire plusieurs mois à l'avance, notamment lorsqu'une chorégraphie est produite par les amis du collège. Le rite revêt une forme d'exceptionnalité dans le parcours de vie d'une jeune fille, car il marque un passage de l'enfance vers une étape ouverte à l'âge adulte féminin. Dans le cas du Mexique, Gerdien Steenbeeck analyse la célébration comme l'étape où "les années de l'enfance sont définitivement terminées et la fille doit maintenant commencer à se comporter comme une femme décente $»^{[10]}$. Si au terme de ce rite, l'entrée dans l'âge adulte reste encore progressif, il est évident que la jeune fille quitte symboliquement l'enfance pour entrer dans une phase biographique où le mariage et la vie de mère de famille et épouse deviennent envisageables. Un changement de statut est donc rendu visible à travers cette cérémonie pour celle qui en est la protagoniste, en lui définissant et instituant un rôle nouveau dans la société.

La célébration aux contours traditionnels de la quinceañera obéit à un ensemble de séquences ritualisées, de codes et d'usages répétés par la coutûme, dans le cadre d'une mise en scène familiale de présentation de la jeune femme à la société. L'organisation et le déroulement de la cérémonie servent également à rassembler la parenté élargie comme les oncles, cousins et grands-parents, ainsi que la communauté «proche » du cercle familial comme les amis des parents et des enfants. Le rite symbolise ainsi pour la jeune fille l'appartenance à un groupe qui dépasse les liens familiaux qui en constituent cependant le noyau central. On peut interpréter cette cérémonie, d'abord, comme un rite identitaire de confirmation d'appartenance dans la mesure où il met en scène l'affiliation de la jeune fille à sa famille, un aspect renforcé par le choix du lieu qui est souvent le domicile parental. Ensuite, la présence de personnes qui ne font pas partie de la famille mais qui y sont liées par des liens d'affinité incarne l'inscription de la jeune fille dans une communauté plus large. La fonction du rite est donc également de construire un réseau social plus large que la famille restreinte ou élargie, c'est-à-dire une ouverture sur la société décisive à terme dans la quête d'un mari. À cet égard, l'habillement de la jeune fille avec

[9] Jacobijn OLTHOFF, op. cit.

[10] Gerdien STEENBEECK, « The years of childhood are definitively over and the girl now has to start behaving like a decent woman. » in Jacobijn OLTHOFF, op. cit., p.128. 
une robe spécialement acquise, voire confectionnée pour la cérémonie, sa couleur blanche ou dans des tons pâles, annonce avec subtilité son mariage. Elle entre alors symboliquement dans un âge de la vie où les noces sont envisageables. Pour renforcer ces allégories, tout ce qui concourt à une émotion collective comme l'entrée sur scène en compagnie du père ou la prise de parole solennelle de la jeune fille et de ses parents est prisé lors du déroulement de la célébration.

Enfin, il est courant que l'ensemble de la cérémonie et de la fête fasse l'objet d'une utilisation continue d'appareils photographiques par les membres de la famille ou par un photographe professionnel engagé pour l'occasion, ainsi que d'enregistrements filmiques partagés ensuite entre la famille et les amis. L'utilisation systématique de ces technologies confère une dimension d'exceptionnalité à la cérémonie et inscrit le rite dans une sorte de temps long et indéfini qui dépasse la seule journée de la célébration. Il peut par la suite être revisualié et remémoré, voire diffusé sur Internet.

\section{Une mise en exergue de l'identité féminine}

Une caractéristique fondamentale du rite des 15 ans est qu'il s'agit d'une célébration strictement réservée aux filles. Il est par conséquent établi une différenciation radicale en termes de genre, dans la mesure où les garçons sont exemptés d'un rite de passage au moins similaire qui leur serait associé. La célébration des 15 ans est une cérémonie au cours de laquelle l'identité de genre féminine est mise en valeur de façon très prononcée. La longue robe de la jeune fille, sa coiffure conventionelle (cheveux longs, le plus souvent détachés), le maquillage soigné et répondant à des critères définis de beauté, la démarche mesurée, la mise en valeur de sa silhouette ou le port de bijoux attirant l'attention du public, voire, dans les cérémonies les plus traditionnelles, d'une couronne sont autant d'éléments mettant en valeur l'identité féminine de la jeune fille. L'usage de ces objets et la manière de se présenter à la société à partir de symboles féminins servent à rompre avec l'enfance et confirment leur entrée dans un âge nouveau renvoyant à la maturité de leur statut de femme adulte.

"En ce qui me concerne, ça a été archi traditionnel : j'ai eu une longue robe, dessinée par moi-même et faite sur mesure, j'étais coiffée de façon très élégante, avec un maquillage léger. Même si je jouais au basket à cette époque et que ce n'est pas un sport franchement féminin, j'ai vécu LA célébration des 15 ans la plus féminine possible qui correspondait totalement à ce qui devait se faire. » (Sandra, 24 ans).

La célébration des 15 ans est un rite sexué, car il ne concerne que des filles de cet âge. L'expérience de cet événement est circonscrit au sexe féminin et participe à la construction socioculturelle d'une différence entre les sexes à l'âge même où s'affirment davantage les identités sexuelles. Ce rite de passage accompagne parallèlement les changements biologiques d'une jeune fille et célèbre implicitement sa sortie de la puberté. Son entrée dans la catégorie des femmes adultes induit sa capacité dorévanant à avoir des enfants et être sexuellement active, ce qui va de pair avec l'annonce tacite du mariage évoquée plus haut. La cérémonie exprime et rend donc visible le changement de statut d'enfant fille à celui d'adulte femme entrant dans une phase longue de préparation au mariage. En ce sens, le rite des 15 ans recouvre une fonction séparatrice sur le plan biologique et marque un point de non-retour quant aux caractéristiques féminines prépubères. 
Enfin, s'il est empiriquement avéré que la cérémonie du quinzième anniversaire est sexuellement discriminante, elle n'exclut pas pour autant la présence des garçons ayant le même âge que la jeune fille, y compris, dans certains cas, de son petit ami lorsque celui-ci est accepté par la famille. Ceux-ci participent aux chorégraphies mises en scène et dansent autour d'elle en jouant un rôle de faire-valoir vis-à-vis de la protagoniste de la cérémonie. Nous verrons plus loin que la participation des garçons est moins régulière dans les formes modernes de célébration des 15 ans.

\section{L'apparition de formes altermatives et «modernes» de célbbration}

\section{Des formes de recréation rituelle de célébration des 15 ans}

En parallèle à la cérémonie traditionnelle des 15 ans étudiée dans la première partie, se sont développés depuis environ une dizaine d'années des modes de célébration profondément distincts choisis délibérement par les jeunes filles en concertation avec leurs parents :

"Je suis partie en voyage en Europe pendant le mois de mon anniversaire. J'ai été enchantée. Ça me paraissait beaucoup mieux de voyager que de célébrer le traditionnel quinceañero par une fête. Bien évidemment, ça a été un moment important dans ma vie, pas pour me sentir plus femme mais pour tout ce que j'ai connu au cours de ce voyage. » (Andrea, 25 ans).

"Pour mes 15 ans, je n'ai pas fait de grande fête comme cela se fait traditionnellement à Lima. J'ai fait une petite fête avec mes amies les plus intimes. Nous avons bu de l'alcool, nous avons mangé des amuse-gueules et quand nous étions un peu saoules, elles m'ont fait danser comme dans une cérémonie traditionnelle. C'était vraiment très drôle. » (Diana, 24 ans).

"I'ai fait une fête dans un local mais pas quelque chose de tapageux du tout. Ma robe et celle de mes copines n'avaient rien des dentelles et étaient beaucoup plus courtes et modernes. Les garçons étaient habillés avec un pantalon et une chemise. Il n'y avait aucun protocole comme celui de la fille qui apparaît avec son père. C'était plus que tout une fête entre jeunes. » (Arlette, 33 ans).

Les trois témoignages qui précèdent, recueillis auprès de liméniennes d'une vingtaine d'années, illustrent les manifestations nouvelles de célébration du quinzième anniversaire remplaçant pour certaines jeunes filles la cérémonie traditionnelle. Il ne s'agit pas d'une dé-ritualisation de la célébration des 15 ans mais d'une recréation du rite selon les attentes nouvelles des jeunes filles ainsi que leurs goûts motivés par la recherche d'originalité et de correspondance avec la mode en cours. Dans cet ensemble, les voyages vers les États-Unis et l'Europe se révèlent être les projets les plus prisés par les jeunes filles. Si, de prime abord, ces modalités de célébration s'écartent sensiblement des normes traditionnelles, les adolescentes identifient ces voyages et autres fêtes entre amis comme des formes de célébration de leur quinzième anniversaire, au même titre que les jeunes filles qui restent dans la lignée des cérémonies traditionnelles. Aucune transgression n'est donc associée à leur choix de ne pas suivre les règles ordinaires de célébration de la quinceañera. Le consentement de leurs parents joue un rôle important dans ce sens. Tout en considérant toujours le passage du quinzième anniversaire comme un âge charnière symbolisant 
la fin de l'enfance, nombre de parents préferrent que leurs filles marquent cet événement de façon plus profitable, notamment par un voyage aux États-Unis pour améliorer leur maîtrise de l'anglais. Dans plusieurs témoignages, on observe également une double célébration du rite, l'une traditionnelle et l'autre dépossédée de tout protocole :

"Mes parents ont organisé deux déjeuners : un premier familial chez ma grand-mère où ils ont invité tous mes oncles et cousins. C'était une grande fête avec un chapiteau et un buffet. Le second a été un déjeuner avec mes meilleures copines du collège dans un restaurant. »(Cristel, 22 ans).

Le rite de célébration des 15 ans se réinvente donc en devenant plus hétérogène et en mettant de côté le déroulement cérémoniel attaché à la culture catholique (longue robe, valse, etc.) et l'intervention prépondérante de la famille.

\section{Un effacement de la famille}

Les formes récentes de célébration des 15 ans renvoient aux fêtes entre copines et aux voyages à l'étranger. Les premières, plus courantes, se déroulent au domicile de la jeune fille, de l'une de ses amies ou dans une salle louée par les parents avec l'appel d'un groupe musical ou d'un disc-jockey. Ce type de célébration, de plus en plus courant, s'observe surtout dans les familles de classe moyenne peu ou pas attachées à la religion catholique. La cérémonie familiale est alors remplacée par une soirée entre copines du même âge (incluant variablement la présence de garçons), mettant ainsi à l'écart la parenté et la famille élargie au profit d'une fratrie élective, et non consanguine, basée sur des affinités horizontales de même sexe, qui met de côté les adultes. Dans le cas des voyages à l'étranger ou à l'intérieur du pays qui sont la manifestation alternative de célébration des 15 ans, la famille n'est pas nécessairement conviée au déplacement. Les parents financent le voyage mais ils laissent généralement leur fille partir avec d'autres copines, ce qui constitue d'ailleurs sa première expérience d'autonomie loin du domicile familial :

«Moi, je suis partie plusieurs jours en voyage avec une amie sur la péninsule de Paracas.

C'était mon premier voyage seule, c'est-à-dire sans ma famille. Ça m'a beaucoup plu, car nous étions seules et même si nous avons pas fait une grande fête, nous avons profité du fait d'être indépendantes pendant ces quelques jours. »(Carla, 24 ans).

On retrouve dans cet exemple la dimension d'acte séparateur du rite des 15 ans. Les filles qui optent en effet pour un voyage avec des amies font l'expérience d'une première séparation physique de leur famille et apprennent à se gérer de façon indépendante. En ce sens, cette forme de célébration des 15 ans rejoint une modalité de rite de passage ou initiatique qui accompagne le changement de statut d'une jeune fille désormais apte à se prendre en charge avec autonomie pendant au moins la durée d'un voyage.

En comparaison aux expressions traditionnelles de célébration du quinzième anniversaire où tous les membres de la famille élargie sont rassemblés au cours de la cérémonie, ces cas montrent un affaiblissement tangible de la place de la parenté. Le rite des 15 ans perdure, certes, mais il se transforme et se réinvente à partir de nouvelles pratiques plus individuelles et juvéniles. Ses manifestations contemporaines, non majoritaires mais de plus en plus fréquentes dans les classes moyennes et aisées, montrent que l'entourage familial perd de son emprise sur cet événement auprès des jeunes filles, au profit des groupes de pairs. 
«Certaines traditions de la célébration se sont perdues. Par exemple, avant, une danse de la jeune fille avec son père et le garçon qui lui plaisait était immanquable, mais maintenant cette danse a été remplacée par des chorégraphies en groupe préparées par les amis, et répétées à l'avance. Par ailleurs, il ne s'agit plus d'une fête aussi solennelle, ce qui peut se remarquer dans l'habillement: les robes ne sont plus aussi longues ou «de princesse », mais courtes et à la mode. De plus, dans les célébrations d'avant, il y avait des invités adultes comme les oncles et les parents d'amis, mais maintenant les fêtes sont des événements presque uniquement entre jeunes. » (Cristel, 22 ans).

Ce phénomène de recréation de la quinceañera n'a pas été observé par Jacobijn Olthoff qui a centré son travail ethnographique sur la vie des jeunes filles dans les quartiers populaires de Lima qui rassemblent les classes sociales où le rite tend majoritairement à se maintenir dans sa forme traditionnelle ${ }^{[11]}$.

\section{Un signe distinctif entre classes sociales}

Parmi les témoignages recueillis, on observe que les jeunes filles qui choisissent de célébrer leur 15 ans par un voyage ou une fête importante entre copines appartiennent à des milieux socioéconomiques plus élevés. L'investissement dans un voyage à l'étranger représente une somme de plusieurs centaines, voire milliers de dollars dont peu de ménages sont en mesure d'assurer le coût. Le choix de ce type de célébration s'apparente donc à un accès à une forme d'élite socioéconomique. Il vise à confirmer et à montrer, non seulement pour la jeune fille mais aussi pour sa famille, un statut social reconnu dans une société péruvienne et liménienne fortement inégalitaire et hiérarchisée entre couches sociales ${ }^{[12]}$. La pratique du voyage à l'occasion du passage des 15 ans est également un moyen pour montrer à l'entourage de la jeune fille qu'elle-même et sa famille sont intégrées dans la globalisation et qu'elles possèdent des capacités de mobilité géographique et d'insertion internationale réservées aux secteurs sociaux les plus dotés en capital économique de Lima.

Parallèlement aux voyages à l'étranger, destinés à une certaine élite, les filles qui organisent avec l'aide de leur famille des fêtes imposantes empruntent des styles de vie et des expériences culturelles se rapprochant des pays occidentaux et en premier lieu des États-Unis.

"Certaines fêtes des 15 ans ont pris dernièrement une importance particulière, très éloignée de la tradition. Il n'y a plus de valse avec le père, de choix du galant. Maintenant, il existe des fêtes où tout doit être plus grand et plus ostentatoire. Elles ressemblent plus que tout aux programmes de MTV ${ }^{[13]}$. » (María, 23 ans).

Ces pratiques novatrices de célébration du passage des 15 ans tendent à se démarquer des traditions de la culture de masse de la quinceañera, liées aux couches populaires majoritaires dans le pays. On peut même parler d'un rejet de la culture populaire de la part d'une frange menue de la population liménienne qui adopte des expériences culturelles venues d'ailleurs. La préférence pour ces formes « modernes » de célébration du quinzième anniversaire pour les filles est une manière de

[11] Jacobijn OLTHOFF, op. cit.

[12] DESCO, La desigualdad en el Perú: situaciones y perspectivas, Lima, Perú Hoy, nº 8, 2005.

[13] MTV (Music Television) est une chaîne de télévision américaine présentant un programme intitulé « My Super Sweet Sixteen » ( «on incroyable anniversaire » en français) racontant la vie de riches adolescentes préparant et célébrant leurs fêtes d'anniversaire d'âge d'or (16 ans aux États-Unis). 
manifester son appartenance à une classe sociale qui se distingue du reste de la société péruvienne. $\mathrm{La}$ « capacité de produire des pratiques [...] classables et la capacité d'apprécier ces pratiques »[14] contribuent à définir un style de vie propre et à affirmer un statut reflétant une position élevée dans la structure sociale de Lima. Le rite initiatique du quinzième anniversaire comme événement de sortie de l'enfance se réinvente mais il n'est plus question comme dans les cérémonies traditionnelles d'une entrée formalisée dans l'âge adulte féminin et de préparation implicite au mariage.

\section{LL'émergence de la jeunesse comme étape de transition entrel 'enfance etl'âğe adultte}

\section{Une résistance du rite}

L'une des observations majeures de l'enquête réalisée sur la célébration des 15 ans pour les jeunes péruviennes est qu'il s'agit d'un rite qui n'a pas perdu de son importance ces dernières années. Il s'est au contraire adapté à des formes plus modernes pour perdurer. Le récit de cette célébration reste pour les femmes d'une trentaine, quarantaine ou cinquantaine d'années une évocation remplie d'émotion et de nostalgie. Cet événement est donc fondateur dans le parcours de vie des jeunes filles. Que le témoignage relate une célébration aux contours traditionnels, une fête entre copines ou un voyage aux États-Unis, les filles identifient cette célébration comme un rite de passage accompagnant leurs changements biologiques et statutaires. À l'instar de nombreux rites, l'aspect le plus décisif n'est certainement pas le déroulement en soi de la célébration mais le souvenir que celui-ci laisse dans la vie de la jeune fille et le sentiment de partager avec ses pairs une expérience commune structurant leur identité féminine.

"Je n'ai pas ressenti un changement si important dans ma vie après cette célébration.

Par contre, je me souviens qu'après la fête, je pouvais en parler en permanence avec mes copines. »(Consuelo, 24 ans).

La même analyse est possible sur le remplacement d'une fête par un voyage. Ce n'est pas tant le voyage dans sa durée qui symbolise la sortie de l'enfance mais l'expérience de celui-ci pour la jeune fille en termes d'autonomie et d'ouverture hors de l'univers domestique. L'intériorisation de ce vécu inédit participe à un changement progressif de statut et s'installe, comme événement biographique majeur, dans le « temps discret » des jeunes filles et leur « calendrier privé » ${ }^{[15]}$. À cet égard, tout événement comporte une face objective - « ce qui survient de manière singulière dans une situation donnée » (une fête entre copines, un voyage à l'étranger) - et une face subjective - « ce qui subsiste et insiste dans le monde subjectif et intersubjectif» (le souvenir entre amies) - qui seul est en mesure de donner un sens au rite et au changement de statut à l'œuvre ${ }^{[16]}$. Que sa forme soit traditionnelle ou moderne, et quoique dépossédée de son caractère religieux, la célébration des 15 ans ne déprécie pas

[14] Pierre BOURDIEU, La distinction. Critique sociale du jugement, Paris, Minuit, 1979, p. 190.

[15] Michèle LECLERC-OLIVE, « Enquêtes biographiques entre bifurcations et événements. Quelques réflexions épistémologiques » in Marc BESSIN, Claire BIDART, Michel GROSSETTI (dir.), op. cit., pp. 329-346.

[16] Catherine NÉGRONI, « Ingrédients des bifurcations professionnelles : latence et événements déclencheurs » in Marc BESSIN, Claire BIDART, Michel GROSSETTI (dir.), op. cit., pp. 176-183. 
sa dimension rituelle. Le rite se transforme seulement, dans ses manifestations contemporaines, vers plus d'individualisme et ne signifie plus une entrée dans l'âge adulte féminin.

\section{Une sortie de l'enfance pour un passage à la jeunesse}

La diversité que prennent les célébrations des 15 ans pour les jeunes filles liméniennes indique que leur biographie se complexifie. Le passage à l'âge adulte cesse progressivement d'être prédéterminé et synchronisé par la cérémonie du quinzième anniversaire, notamment dans ses formes les plus récentes. Si la sortie de l'enfance est confirmée dans la concrétisation de ce rite, les jeunes filles qui sont très majoritairement étudiantes et toujours célibataires, qui vivent au domicile de leurs parents et projettent d'entamer des études après le collège, entrent dans un entre-deux étalé entre l'enfance et l'âge adulte : la jeunesse. Cette étape émerge au Pérou comme période charnière dans les parcours de vie des jeunes filles. Le groupe de pairs, le collège et l'université en perspective remplacent la famille étendue comme principaux espaces de socialisation. Les évolutions récentes et prononcées de célébration des 15 ans observées pour les filles convergent vers cette tendance. Elles indiquent un allongement de la période de transition entre l'enfance et le statut d'adulte ainsi que l'émergence consécutive de la jeunesse, en premier lieu dans les villes du pays. Ce phénomène n'est pas exclusif au Pérou et à l'Amérique latine mais se remarque également dans des pays comme le Cameroun où est fait état d'un « entre-deux flou des jeunes citadins africains $»^{[17]}$. Sans faire explicitement référence à un « entre-deux » et à une entrée dans la jeunesse, Gerdien Steenbeck observait déjà dans les années 1990 à propos de la jeune fille célébrant la quinceañera au Mexique, qu'elle entrait dans un âge où elle n'était ni une fille ni une femme, mais les deux en même temps ${ }^{[18]}$.

30 L'événement fondateur de la quinceañera se maintient en tant que jalon et expression symbolique de la fin de l'enfance mais il s'avère insuffisamment pertinent pour analyser l'entrée des filles dans l'âge adulte. Ce constat laisse apparaître la catégorie de jeunesse, étape aux limites floues et dont la définition varie couramment d'un pays à l'autre. L'avènement de la jeunesse est concomitant à la recomposition des rites de passage, l'allongement de la durée des études supérieures, le report des transitions professionnelles et la prolongation du temps précédant la mise en couple qui tendent à repousser toujours plus tard l'âge d'accès à un plein statut adulte ${ }^{[19]}$. L'observation de l'évolution de certains indicateurs démographiques et sociaux aide à mieux cerner et démontrer l'émergence de cette étape de la vie au Pérou.

\section{Un reflet d'indicateurs démographiques et sociaux}

Les transformations récentes du rite du quinzième anniversaire pour les jeunes liméniennes entrent en consonnance avec les évolutions démographiques et sociales contemporaines de la société péruvienne, notamment en ce qui concerne la population féminine des villes. D'abord, le taux de natalité de la population de 15 à 19 ans a baissé de $79 \%$ à $59 \%$ entre 1986 et $2006^{[20]}$,

[17] Anne CALVÉS, Michel BOZON, Alioune DIAGNE, Mathias KUÉPIÉ, « Le passage à l'âge adulte : repenser la définition et l'analyse des "premières fois" », in Philippe ANTOINE, Éva LELIÈVRE, États flous et trajectoires complexes. Observation, modélisation, interprétation, Paris, INED, 2006, pp. 137-156.

[18] « She is neither a girl, nor a woman and both at the same time » in Gerdien STEENBEEK, op. cit., p. 93.

[19] Olivier GALLAND, Sociologie de la jeunesse, Paris, Armand Colin, 2001.

[20] INEI, Perú: encuesta demográfica y de salud familiar 2004-2006, Lima, 2007. 
sous l'effet d'un usage plus généralisé des méthodes contraceptives qui se sont démocratisées, notamment dans les villes et auprès des classes moyennes ${ }^{[21]}$. Ce taux a également diminué au cours de la même période pour les 20-24 ans (184 \%o à $125 \%$ ), les 25-29 ans (199\%o à $121 \%$ ) et les 30-34 ans (161\%o à $107 \%$ ). Ces tendances se retrouvent dans un taux de fécondité qui a baissé de 4,3 à 2,6 enfants par femme pendant cette période (3,1 à 2,1 enfants par femme dans les zones urbaines). En ce qui concerne l'âge moyen de naissance du premier enfant en zone urbaine, celui-ci est passé de 22,8 ans pour les femmes entre 45 et 49 ans en 2006 à 24,1 ans pour celles entre 25 et 29 ans la même année. Ce retardement progressif de la naissance du premier enfant s'accentue à mesure que l'on avance dans les couches socio-économiques les plus riches de la population péruvienne urbaine et parmi les niveaux d'éducation les plus élevés ${ }^{[22]}$. Par rapport à l'âge moyen de première union, des tendances similaires apparaissent. Pour les femmes de 45 à 49 ans en 2006, celui-ci était de 22,4 ans contre 23,8 ans pour celles de 25 à 29 ans. De la même manière, les différences s'accentuent davantage pour une même génération, par exemple pour les femmes de 25 à 29 ans, entre le niveau d'éducation le plus faible (18 ans en moyenne) et le plus élevé (26,4 ans en moyenne), à l'image des deux extrémités en termes de couches socio-économiques (18,5 contre 27,6 ans en moyenne $)^{[23]}$. On pourrait enfin ajouter à ces données l'allongement d'ensemble de la scolarité et des études supérieures (techniques ou universitaires) pour les jeunes filles péruviennes, en particulier pour celles résidant dans les villes du pays ${ }^{[24]}$.

Ces quelques chiffres indiquent que les principaux jalons marquant l'entrée dans l'âge adulte féminin comme la naissance du premier enfant ou la première union sont repoussés dans le temps, notamment dans les couches socio-économiques intermédiaires et les plus élevées. Ces évolutions du statut des femmes dans la société péruvienne et son maintien prolongé dans la jeunesse renvoient à une modernisation du pays faisant suite à la transition démographique des années 1960 et 1970.

Les résultats de l'enquête montrent que si le rite du quinzième anniversaire pour les filles subsiste au Pérou dans sa dimension traditionnelle, celui-ci évolue, notamment dans les classes moyennes et aisées, vers des formes de festivité écartant le rôle de la famille au bénéfice des groupes de pairs et des voyages à l'étranger. Cette diversification du rite indique en substance que l'abandon des traditions ne s'accompagne pas d'une « dé-ritualisation » de la société. Des phénomènes de recréation font jour et donnent lieu à de nouveaux types de célébration reflétant les évolutions contemporaines de la société et de la culture auxquelles ce rite appartient.

Du point de vue méthodologique, l'article a souligné l'importance de l'interdisciplinarité dans les sciences sociales. Si l'étude des rites est traditionnellement associée à l'ethnologie et l'anthropologie, la sociologie a permis le recueil de témoignages et une analyse de la célébration des 15 ans dans une perspective biographique alors que la démographie a rendu possible la mise en perspective des résultats avec des données quantitatives.

[21] INEI, op. cit.

[22] INEI, $o p$. cit.

[23] INEI, op. cit.

[24] Laure PASQUIER-DOUMER, « La evolución de la mobilidad escolar intergeneracional en el Perú a lo laro del siglo XX », Bulletin de l'Institut Français d'Études Andines, 31, 3, 2002, pp. 429-471. 
Pour approfondir davantage les évolutions contemporaines de la cérémonie des 15 ans au Pérou et dans d'autres pays d'Amérique latine, il serait intéressant de mener une enquête à la fois quantitative et qualitative, sur un échantillon plus vaste, afin de comparer le maintien ou les réélaborations des coutumes liées à ce rite entre classes sociales et milieux culturels (familles de migrants en provenance de la région andine ou amazonienne, familles transnationales, etc.). Une étude de cette nature permettrait en effet de confirmer à une plus grande échelle les tendances esquissées dans cet article et analyser plus finement encore la conservation des coutumes et les recréations contemporaines de ce rite de passage au Pérou. 